\title{
Mirror illumination and spillover measurements of the Atacama Cosmology Telescope
}

\author{
Patricio Gallardo ${ }^{a, b}$, Rolando Dünner ${ }^{a, b}$, Ed Wollack ${ }^{c}$, Fernando Henriquez ${ }^{d}$ and Carlos \\ Jerez-Hanckes $^{d}$ \\ aDepartamento de Astronomía, Facultad de Física, Pontificia Universidad Católica de Chile, \\ Vicuña Mackenna 4860, Santiago, Chile \\ ${ }^{b}$ Centro de Astro Ingeniería, Pontificia Universidad Católica de Chile, Vicuña Mackenna 4860, \\ Santiago, Chile \\ ${ }^{c}$ Code 553/665, NASA/Goddard Space Flight Center, Greenbelt, MD 20771, USA \\ ${ }^{d}$ Departamento de Ingeniería Eléctrica, Escuela de Ingeniería, Pontificia Universidad Católica \\ de Chile, Vicuña Mackenna 4860, Santiago, Chile
}

\begin{abstract}
The Atacama Cosmology Telescope (ACT) is a $6 \mathrm{~m}$ telescope designed to map the Cosmic Microwave Background (CMB) simultaneously at $145 \mathrm{GHz}, 220 \mathrm{GHz}$ and $280 \mathrm{GHz}$. The receiver in ACT, the Millimeter Bolometer Array Camera, features 1000 TES bolometers in each band. The detector performance depends critically on the total optical loading, requiring the spillover contributions from the optics to be minimal. This inspired the use of a cold Lyot stop to limit the illumination of the primary and the use of guard rings surrounding the primary and secondary reflectors. Here, we present a direct measurement of the illumination aperture for both reflectors and of the attenuation level outside the main optical path. We used a $145 \mathrm{GHz}, 1 \mathrm{~mW}$ source and a chopper wheel to produce a time-varying signal with a broad beam profile. We sampled the response of the camera for different locations of the source, placed in front and beside the primary and secondary mirrors. The aperture of the primary was measured to be $5.72 \pm 0.17 \mathrm{~m}$ in diameter ( $95 \pm 3 \%$ of its geometrical size), while the aperture of the secondary yielded $2 \pm 0.12 \mathrm{~m}$ in diameter. Both apertures are consistent with the optical design. Comparing to previous measurements of the beam solid angle from planet observations, we estimate an optical efficiency of $72.3 \pm 4.8 \%$. We found that the attenuation outside the primary aperture was $-16 \pm 2 \mathrm{~dB}$, which is below the theoretical expectations, and $-22 \pm 1 \mathrm{~dB}$ outside the secondary aperture, which is consistent with simulations. These results motivated the extension of the baffles surrounding the secondary mirror, with the following reduction in detector optical loading from $2.24 \mathrm{pW}$ to $1.88 \mathrm{pW}$.
\end{abstract}

Keywords: ANTENNAS, SUB-MM ASTRONOMY, INSTRUMENTATION

\section{INTRODUCTION}

The Atacama Cosmology Telescope (ACT) is a 6-meter dish millimeter wave telescope located at Cerro Toco in northern Chile at and elevation of $5200 \mathrm{~m}$. It is designed to measure the Cosmic Microwave Background (CMB) radiation with arcminute resolution, sampling multipoles up to $\ell \approx 10^{4}$ and producing high sensitivity maps . ${ }^{1}$ The camera in ACT between the years 2007 and 2010 was called MBAC. It was designed to observe simultaneously in three bands centered at $145 \mathrm{GHz}, 220 \mathrm{GHz}$ and $280 \mathrm{GHz}$, with bandwidths of approximately $30 \mathrm{GHz}$ for each frequency, each one sampled with a kilopixel TES bolometer array. ${ }^{2}$ These frequency bands were chosen to sample the decrement, null and increment regions of the thermal Sunyaev-Zel'dovich effect in galaxy clusters, ${ }^{3}$ permitting their direct detection from the maps. ${ }^{4,5}$ The observations done during those years covered two long tripes of the sky, one centered at declination -51 deg and the other at the equator, mapping a total of 2000 square degrees in the sky. There is a long list of scientific and technical contributions from this experiment in the areas of cosmology, eariy universe and cluster physics. ${ }^{4-8}$

\footnotetext{
R.D.: E-mail: rdunner@astro.puc.cl; P.G.: E-mail: pngallar@ing.puc.cl
} 


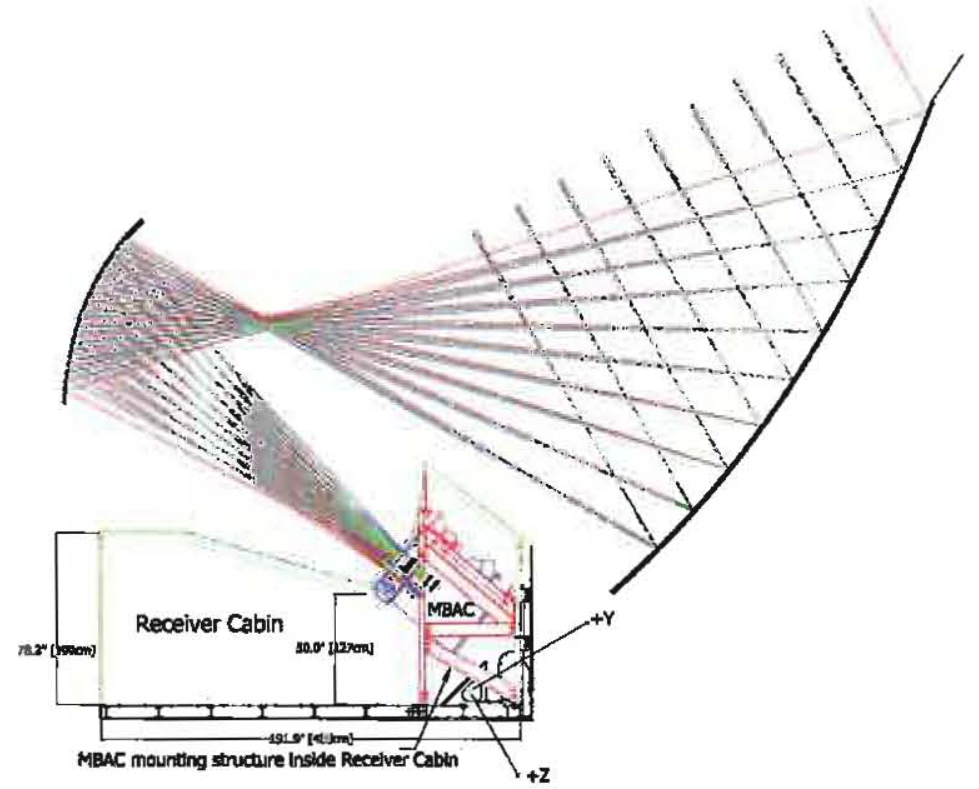

Figure 1: Main optics schematic of the ACT telescope overlaid with colored ray tracing for each of the three frequency bands. The telescope here is pointing to an elevation of $60 \mathrm{deg}$. Courtesy of J. Fowler. ${ }^{1}$

ACT has an off-axis gregorian optical design, with a $6 \mathrm{~m}$ primary reflector followed by a $2 \mathrm{~m}$ secondary reflector, which focuses the light into the camera, as shown in Figure 1. Inside the camera the light is refocused onto the focal plane using a system of refractive elements at different cryogenic temperatures, one for each frecuency band. Each focal plane is populated with $1024,1 \times 1 \mathrm{~mm}$ size, pop-up detectors, coupled directly to the incoming optical signal through a coupling layer for impedance matching. This horn-free configuration has the advantage that it allows a denser detector population in the focal plane $(0.5 f \lambda$ in this case) to achieve a faster mapping speed. One counter side is that each detector has a wide field of view, so a cold Lyot stop is required to reduce the stray radiation coming from outside the optical path to limit the power received by the detectors. ${ }^{9}$ The Lyot stop was designed to limit the illumination of the primary refector, defining the effective aperture of the system. Even with a Lyot stop, light can still diffract on the different components of the optical system and couple in the detectors, producing spillover effects or stray radiation. At this level of sensitivity, optical loading critically limits the performance of the system and needs to be minimized. The total optical loading in the telescope has been measured and it is around $2.6 \mathrm{pW}$, from which $0.7 \mathrm{pW}$ comes from water vapor in the atmosphere at nominal atmospheric conditions. We also expect that the contribution of the ground and surroundings coupling through far sidelobes is less than $0.1 \mathrm{pW}$ given the ground shielding and geometry of the telescope, as published in a companion paper (SPIE 8452-77). To reduce the temperature of the stray radiation coming from around the mirrors, guard rings were built around the primary and secondary mirrors to reflect the rays directly to the sky by artificially extending the collecting area. In 2010 , additional guard rings were installed around the secondary mirror extending their area. The effect was a $14 \%$ reduction of the optical loading of the system.

Several numerical simulations have been done to understand the performance of the optical system. Here we present an attempt to experimentally measure the illumination of the mirrors and quantify the spillover loading, as well as experimentally determine the limits of the main aperture of the telescope defined by the Lyot stop.

In Section 2 we provide some definitions of optical efficiency employed, in Section 3 we explain the method usec to measure the illumination and spillover. In Section 4 we describe the data analysis techniques, in Section 5 we present our results and discussion and in Section 7 we present our conclusions. 


\section{OPTICAL EFFICIENCY}

At longer wavelengths, diffraction effects become important reducing the performance of optical systems. In radioastronomy it is common to speak about the antenna power pattern, $P(\theta, \phi)$, which models the angular distribution of the system's response to a plain wave (point source), normalized such that $P(0,0)=1$ in the forward direction. The effective antenna solid angle is then defined as

$$
\Omega_{A}=\iint_{4 \pi} P(\theta, \phi) d \Omega .
$$

We can define the effective area, $A$, of the telescope as the cross section that the telescope opposes to an incoming radiation flux in the forward direction. It can be shown that, for monochromatic raciation, it satisfies the relation

$$
A \Omega_{A}=\lambda^{2},
$$

where $\lambda$ is the radiation wavelength.

The effective aperture is less than the geometrical aperture, $A_{g}$ (actual size of the collecting area), by a factor of the aperture efficiency factor,

$$
\eta_{A}=\frac{A}{A_{g}},
$$

due to various losses in the optical system. The losses can be caused by blockage (not this case), illumination taper, surface errors in lenses and mirrors, spillover efficiency, among others. All of these effects multiply- each other such that

$$
\eta_{A}=\eta_{\text {block }} \eta_{\text {taper }} \eta_{\text {surf }} \eta_{\text {spill }} \ldots
$$

Point source measurements can be used to determine the antenna power pattern, at least for the main lobe and a few sidelobes at the most, but not for the whole solid angle. The effective antenna solid angle can be divided in the main beam solid angle, $\Omega_{m}$, and the side- and back-lobes solid angle, $\Omega_{l}$, such that

$$
\Omega_{A}=\Omega_{m}+\Omega_{l}=\iint_{\text {main beam }} P(\theta, \phi) d \Omega+\iint_{\text {sidelobes }} P(\theta, \phi) d \Omega .
$$

We can define the main beam efficiency as

$$
\eta_{m}=\frac{\Omega_{m}}{\Omega_{A}},
$$

which can be used to characterize the amount of power that there is in the sidelobes with respect to the main beam.

When determining the telescope efficiency experimentally out of known quantities $\left(A_{g}, \Omega_{m}\right.$ and $\lambda$ ), we can only determine the ratio between the aperture and main beam efficiencies as

$$
\frac{\eta_{A}}{\eta_{m}}=\frac{\lambda^{2}}{A_{g} \Omega_{m}} .
$$

$\mathrm{Bv}$ design the main beam efficiency of the ACT telescope is close to unity. Measurement of the geometrical area, combined with previous measurements of the main beam solid angle from planet observations, can provide useful limits on the aperture efficiency.

\section{METHOD}

To map the illumination of the reflectors and surrounding area, a $145 \mathrm{GHz}$ source was placed at different positions in front and around the primary and secondary mirrors. By comparing the response of the telescope inside and outside the optical path, we expect to be able to quantify the attenuation outside the aperțure defined by the Lyot stop, as well as to determine the edge of the illuminated area. 

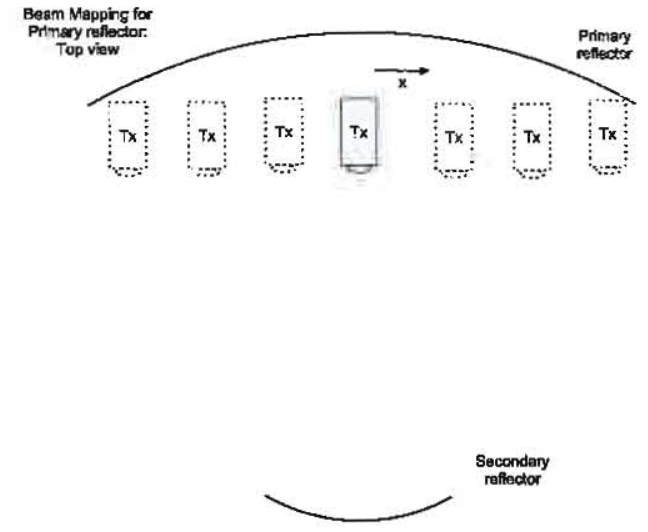

(a) Primary reflertor Beam Map

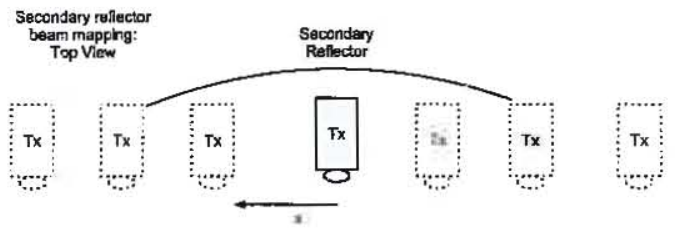

MBAC

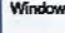

(b) Secondary reflector Beam Míap

Figure 2: Source positions used for primary and secondary beam map. The small ellipse in front of the source represents the source window. Note that the source is maintained in the same orientation while moved horizontally in front of the mirror. As a result, the measurements must be corrected using the antenna pattern of the source, previously measured in the laboratory.

\subsection{Source Design}

The source had to be chopped to modulate the signal above the $1 / f$ noise of the camera and awav from the atmospheric emission fluctuations. In order to make measurements consistent with each other, we designed the source as isotropic as possible. This has the disadvantage that light can be reflected in unknown places within the telescope and add up power to the spillover measurements, so we expect the measured spillover levels to be an upper bound to the level at each point.

A Gunn diode feeding a WR6 waveguide is used as a radiation source for mapping. A rariable attenuator was used to control the output power and to reduce the formation of standing waves inside the waveguide. To provide a wide antenna pattern, we used an open waveguide as feed for the system. A five-blade chopper was placed in front of the feed, modulating the signal at $5 \mathrm{~Hz}$. The whole system was placed inside an aluminum box for protection and thermal control, which was carried out with a heater and a closed loop control system. The box had a $7 \mathrm{~cm}$ window opening to let the radiation come out, while ECCOSORB* was used to dump the radiation that could bounce inside the box. The source was characterized in the laboratory and simulated using numerical methods to understand its stability and output power profile. The latter is fundamental to deconvolve the resuits and compensate the properties of the source. More details of the source properties and characterization work is provided in a companion paper (SPIE 8452-77).

\subsection{Experimental Setup}

Our mapping strategy consisted in sampling the response of the telescope for source positions along horizontal lines across the primary and secondary mirrors, extending to regions outside the main optical path. Figure 2 contains a schematic diagram with the locus of the source in front of each mirror.

To hold the source at these positions, it was mounted on a rail attached to a support that could be held to a ladder placed in front of each mirror, as shown in Figure 3. With this setup, the source could be moved along the rail and the rail could be slid horizontally through the ladder mount to extend the reach to either side. In the case of the primary mirror, the ladder could also be moved horizontally on wheels. The ladder in front of the primary was there for all experiments, while the one in front of the secondary was there exclusively for the secondary survey.

\footnotetext{
* rww. eccosorb.com
} 


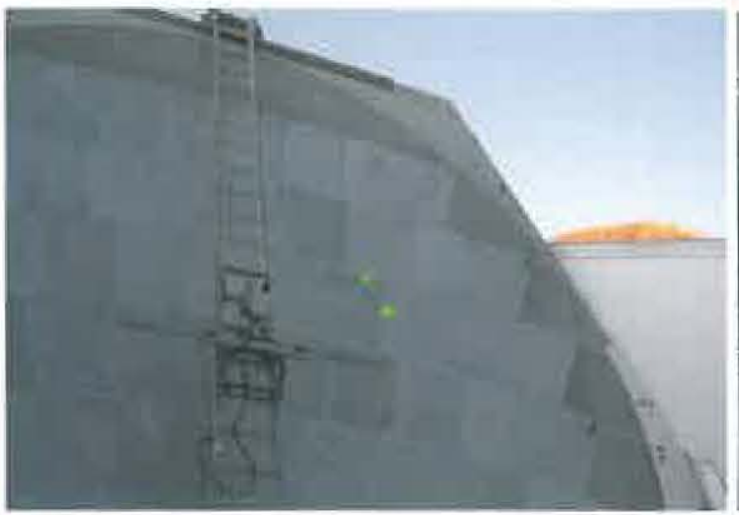

(a) Primary mirror beam map.

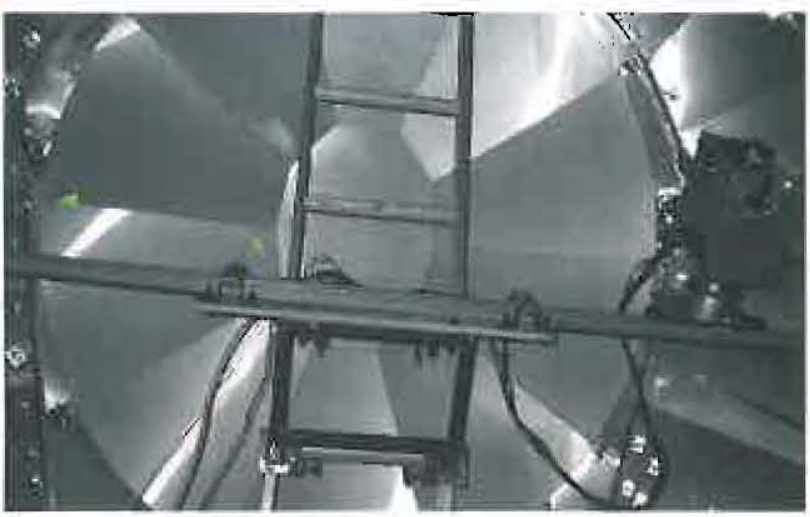

(b) Secondary mirror beam map.

Figure 3: (a) Picture of the source mounted in front of primary mirror for the center survey. Note that the radiation leaving the source needs to hit on the secondary mirror before reaching the camera. (b) Picture of the source mounted in front of the secondary mirror for the center survey. In this case, the radiation goes directly into the camera. The green arrow indicates the direction of the $\mathrm{X}$ axis. The ladder in front of the secondary mirror was not present while sampling the primary mirror.

The position of the source for each measurement was monitored using a Faro Laser Tracker ${ }^{\dagger}$ with a precision of $\approx 5 \mu \mathrm{m}$ and a laser pointer was used to orient it in the forward direction. The temperature of the source was monitored during the experiment, showing variations with a standard deviation of $0.1^{\circ} \mathrm{C}$.

The signal was measured by the camera (MBAC) and stored in RAW, time ordered data (TOD) format, with a sampling rate of $400 \mathrm{~Hz}$. Before performing the measurements, the camera was properly biased using the standard biasing procedure. After that, several tests were done to adjust the source attenuation to avoid saturating the detectors and maximizing their dynamical range.

\subsection{Measurements}

The measurements were done in January, 2011. Unfortunately, the precipitable water vapor (PWV) was high during those nights, increasing the loading on the detectors and saturating many, so less than 600 detectors were available for the measurements.

We performed two horizontal surveys on each of the mirrors, one near the center and one above the center. For the primary mirror, we sampled 27 points across the center and 16 points for the upper survey. In each case the source was first centered in the mirror and aimed towards the center of the secondary mirror. Then it was moved to the other positions witholit changing its orientation, such that the signal is expected to be modeled by the antenna pattern of the source. For the secondary mirror, we measured 13 points across the center and 23 points above the center. Again the source was first centered and aimed towards the center of the camera. window before moving it horizontaily without changing its orientation. The sampling was sparser inside the illuminated aperture than near and outside the edges of the mirrors to help characterize them better. During the measurements the source temperature was stable enough to think that the source power was also stable. Figures 2 and 4 show representations of the locus of the survey points for each mirror.

\section{DATA ANALYSIS}

To estimate the intensity in each detector, we obtain the power spectrum of the signal and search for a peak in a frequency window of $6 \mathrm{~Hz}$ of bandwidth between $2 \mathrm{~Hz}$ and $8 \mathrm{~Hz}$. For the observed points we obtained a SNR ranging from 6 to $28 \mathrm{~dB}$.

\footnotetext{
$\dagger_{\text {พw. faro.com }}$
} 


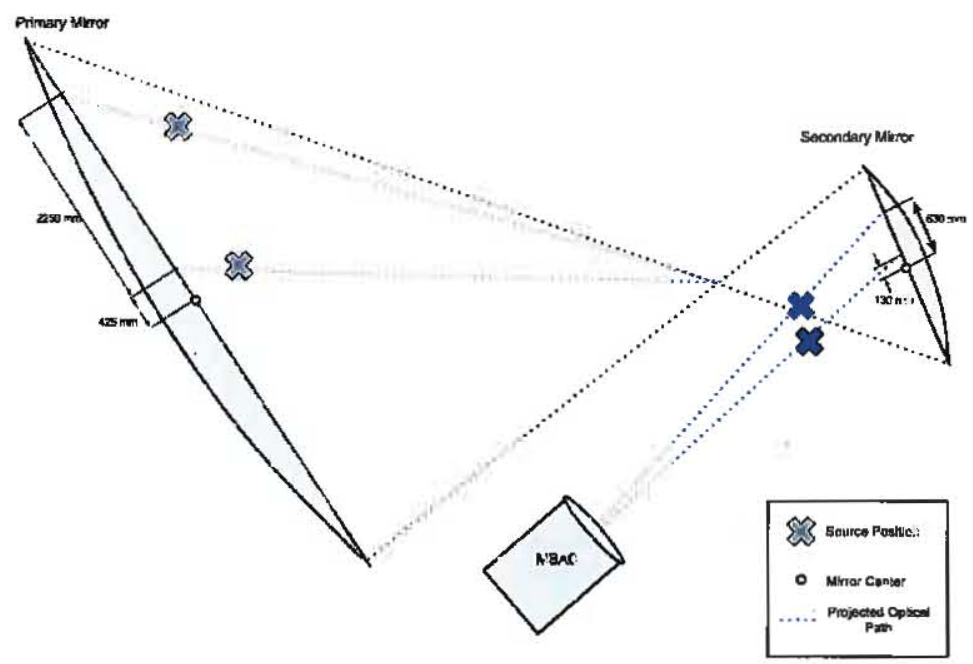

Figure 4: Source position on beam mapping. Blue crosses show the position of the source for each survey. The blue segmented lines show the line of sight from the source to a pivot point (gregorian focus for the primary and camera window for the secondary mirror). Red dots show the center of each mirror.

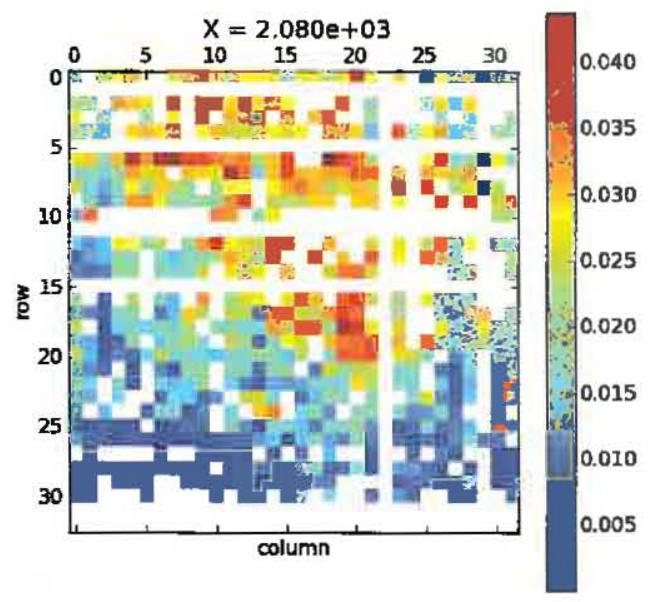

(a)

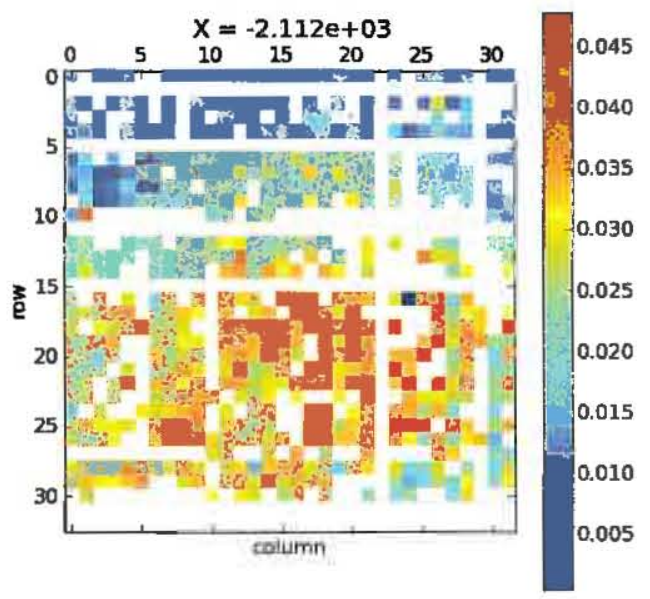

(b)

Figure 5: Focal plane illumination distribution in units of $\mathrm{pW} / \sqrt{\mathrm{Hz}}$ for measurements done on the central primary survey. (a) and (b) show the detected power at $X=2.08 \mathrm{~m}$ and $X=-2.11 \mathrm{~m}$ from the center of the mirror respectively.

Mapping the detector response across the array, we can see that the signal is not uniform, forming wide patches of different intensity, as shown in Figure 5. We used the median of the array as our best estimator for the power at every given point, while the error was estimated using the variance over the array. These were the dominant uncertainties in the measurements.

The positions of the source had to be projected onto the surface of the mirrors to map their illumination. 


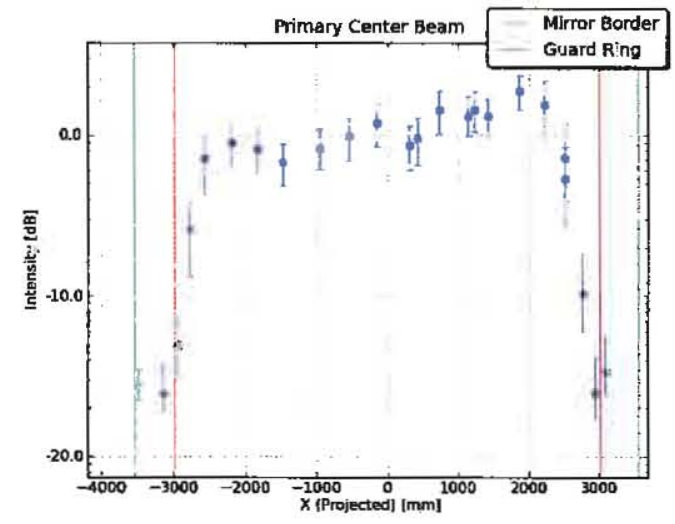

(a) Primary Center

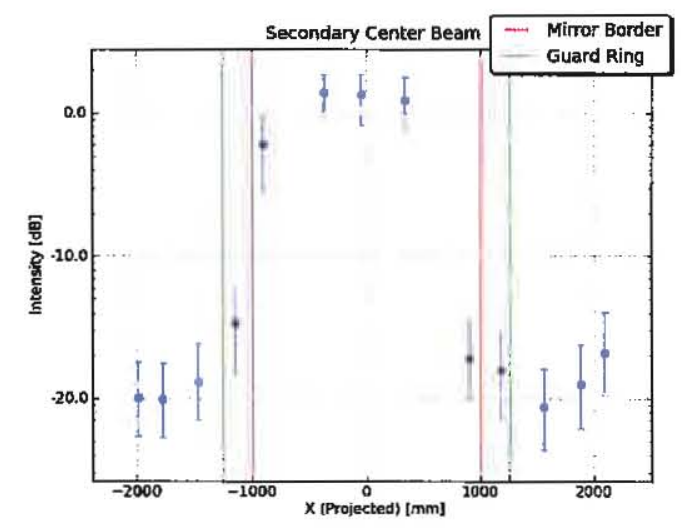

(c) Secondary Center

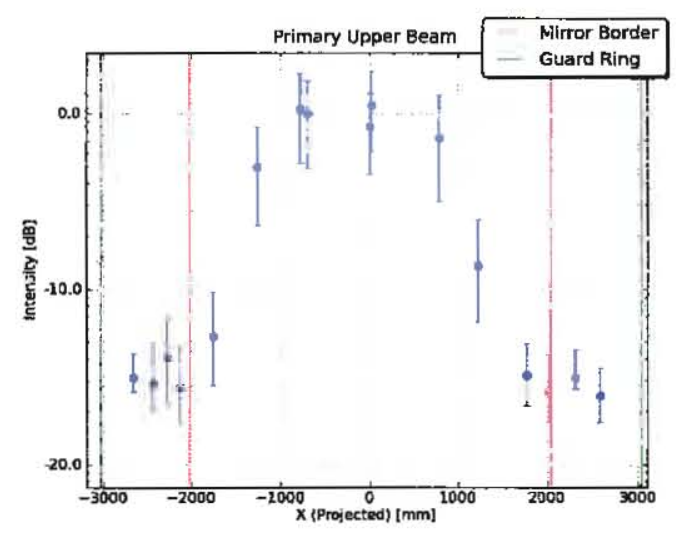

(b) Primary Upper

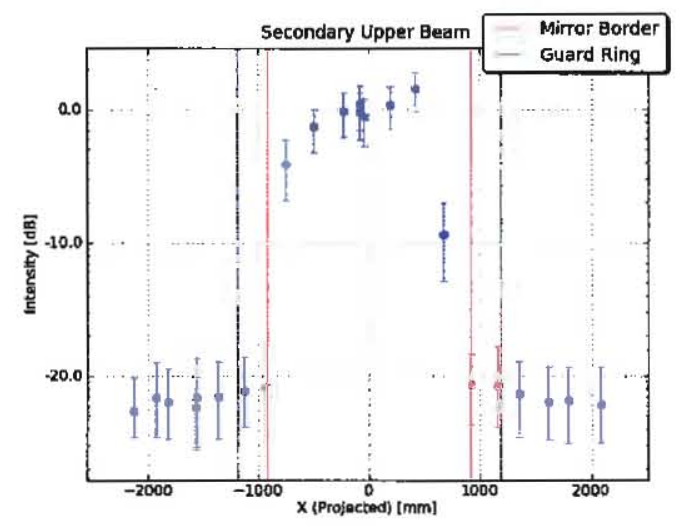

(d) Secondary Upper

Figure 6: Primary and secondary mirror survey results. The $X$ values indicate the position of the source projected onto the surface of the mirror. The values are given in decibels with respect the the centrai value, determined by the average of the four central measurements. The error bars show the $68 \%$ confidence level of the array detector values. The red vertical line indicates the edge of the mirror, while the green line indicates the outer edge of the guard ring.

This was done considering the trajectory of light onto each mirror. For the primary mirror, the gregorian focus was used as a pivot point to calculate the angles, while for the secondary it was assumed that all rays converged at the center of the camera window. Figure 4 explains the geometry of the problem.

\section{RESULTS}

Figure 6 show the results for each of the four surveys. It can be seen that the measuremerts vary by $3 \mathrm{~dB}$ across the aperture. We attribute these differences to the same systematics causing the non-homogenous response of the array and not to a real aperture imbalance, as indicated by the error bars. This adds a $3 \mathrm{~dB}$ uncertainty to the attenuation levei of the spillover, but has a small effect in the determination of the aperture limit.

We arbitrarily defined the edge of the illuminated region where the measured profile reached $-10 \mathrm{~dB}$. Table 1 shows the spillover attenuation for each survey; averaged from all the points in Figure 6 with attenuation below: $-10 \mathrm{~dB}$. In the primary central survey only a few points were measured outside this limit because they were hard to reach with our setup. Still, the attenuation measured in those few points is consistent with the one measured in the upper survey. For the secondary mirror, much higher spiliover levels were measured in the central survey than in the upper survey, for which the results are consistent with a $0.6 \%$ level predicted from simulations. ${ }^{1}$ 


\begin{tabular}{|l|c|c|}
\hline Survey & Spillover Level [dB] & Spillover Level [\%] \\
\hline \hline Primary center & $-15 \pm 3 \mathrm{~dB}$ & $3 \%$ \\
Primary upper & $-15 \pm 3 \mathrm{~dB}$ & $3 \%$ \\
Secondary center & $-17 \pm 3 \mathrm{~dB}$ & $2 \%$ \\
Secondary upper & $-22 \pm 3 \mathrm{~dB}$ & $0.6 \%$ \\
\hline
\end{tabular}

Table 1: Average spillover attenuation measured in each survey. The values correspond to the average of all poiats $-10 \mathrm{~dB}$ in Figure 6 . These values provide an upper bound to the spillover level in those regions, as extra loading could arise from reflections in parts of the telescope structure given the wide antenna pattern of the microwave source used in the experiment.

\begin{tabular}{|lcccc|}
\hline Mirror & $a[\mathrm{~m}]$ & $b[\mathrm{~m}]$ & $D[\mathrm{~m}]$ & $\overline{A_{g}}\left[\mathrm{~m}^{2}\right]$ \\
\hline \hline Primary (design) & & & 5.82 & 26.6 \\
Primary (-10 dB) & $2.86 \pm 0.09$ & $3.20 \pm 0.01$ & $5.72 \pm 0.18$ & $25.7 \pm 1.6$ \\
Secondary (-10dB) & $1.02 \pm 0.05$ & $1.02 \pm 0.05$ & $2.04 \pm 0.70$ & $3.3 \pm 0.3$ \\
\hline
\end{tabular}

Table 2: Summary of the primary and secondary iliumiration parameters. $a$ and $b$ are the mayor and minor axes of an ellipse fitted to the edge of the illuminated surface on the mirrors (measured at $-10 \mathrm{~dB}$ from the central power), $D$ is the effective diameter of the illumination perpendicular to the optical axis, and $A_{g}$ is the illumination area perpendicular to the optical axis, and corresponds to the geometrical area of the telescope.

The extra loading is believed to arise from reflections on the structural surfaces between the camera and the secondary mirror, on top of the receiver cabin in Figure 1, and where we were standing. As mentioned before, we expect these values to represent an upper bound to the real value.

The aperture of each reflector was calculated by fitting an ellipse to the 4 points defining the edge of the illuminated region. Those points were obtained by linearly interpolating the data to find the $-10 \mathrm{~dB}$ crossing. The ellipse was then projected to a plane normal to the opticai path, being in both cases consistent with a circle of ciameter $D_{\text {prim }}=5.72 \pm 0.18 \mathrm{~m}$ for the primary mirror and $D_{s e c}=2.04 \pm 0.70 \mathrm{~m}$ for the secondary mirror. The illuminated area represents the geometrical aperture of the telescope as defined by the Lyot stop inside the camera, which for the primary is $A_{g}=25.7 \pm 1.6 \mathrm{~m}$. The aperture diameter defined by the Lyot stop in the initial optical design of the teiescope was $5.82 \mathrm{~m}$, implying a collecting area of $26.6 \mathrm{~m}^{2}$. Table 2 contains a summary of these results.

\section{DISCUSSION}

This measurement of the geometrical aperture of the telescope can be used to characterize the optical efficiency of the system.

The main beam solid angle has been measured from planet observations by the ACT team. According to Hincks et al. (2010), ${ }^{4}$ based on observations done in 2008 , the beam solid angle for the $148 \mathrm{GHz}$ camera is $\Omega_{m}=218.4 \pm 4.0 \mathrm{nsr}$. Taking the center of the frequency band, we can use $\lambda=2.02 \mathrm{~mm}$ on Equation 2 to calculate a "main beam" effective aperture

$$
A_{m}=\frac{\lambda^{2}}{\Omega_{m}}=18.57 \pm 0.34 \mathrm{~m}^{2} .
$$

This value is clearly lower than the geometrical aperture in Table 2 denoting the loss of efficiency. We can estimate the system efficiency from the ratio of these two quantities (Equation 7):

$$
\frac{\eta_{A}}{\eta_{m}} \approx \eta_{A}=\frac{A_{m}}{A_{g}}=72.3 \pm 4.8 \% .
$$

From numerical simulations done in Zemax using the Huygens method, an optical efficiency of $89.4 \%$ is predicted for the telescope's design configuration. The Huygens method accounts for taper effects, phase errors on the aperture and spillover effects. If we consider that the detectors are square pixels of $1 \mathrm{~mm}$ sides, then we expect that the simulated PSF projected on them would get convolved vith the pixel size, providing another $87.2 \%$ 


\begin{tabular}{|lcc|}
\hline Source & Marginal Efficiency & Accumulated Efficiency \\
\hline \hline Huygens Diffraction & $89.4 \%$ & $89.4 \%$ \\
Detector Convolution & $87.2 \%$ & $80.0 \%$ \\
Surface Errors & $96.6 \%$ & $75.3 \%$ \\
\hline Main Beam (Hincks) & & $72.3 \pm 4.8 \%$ \\
\hline
\end{tabular}

Table 3: Optical efficiency analysis derived from measuring the geometrical aperture of ACT. The Huygens diffraction analysis was done numerically in Zemax and accounts for taper, phase and spillover effects. The efficiency loss produced by the non-negligible size of the detectors is accounted for by convolving the detector size with the optical PSF projected on the focal plane. The surface error efficiency comes from applying the Ruze formula to the measured surface rms of the primary mirror. The total efficiency was obtained using the beam solid angle measured from planet observations in Hincks et al. (2010). ${ }^{4}$

efficiency factor. On top of this, we can consider that the surface errors of the primary mirror have been measured to be $\sigma=30 u \mathrm{~m}$ rms. Applying the Ruze formula, we obtain a surface efficiency is $\exp \left(-(4 \pi \sigma / \lambda)^{2}\right)=0.966$. This leaves us a net efficiency of $75.3 \%$, which is within our measurement errors. Upon consideration of other potential surface errors (e.g., thermal expansion, gravitational deformation, panel gaps, etc) this is considered an upper bound. Table 3 shows a summary of the efficiency budget of the system.

\section{CONCLUSIONS}

We have successfully measured the illumination of the primary and secondary mirrors of the ACT telescope, as well as provided upper bounds to the spillover near the edges of the illuminated areas.

We showed that the spillover level around the primary mirror was less than $-15 \pm 3 \mathrm{~dB}$, while around the secondary it reached less than $-22 \pm 3 \mathrm{~dB}$, which is consistent with the expectations. These non-negligible amounts of spillover justify the use of guard rings around both mirrors to redirect the light to the sky in order to reduce the amount of optical loading on the detectors.

We also provided a measurement of the geometrical aperture of the ACT telescope, which yielded $A_{g}=$ $25.7 \pm 1.6 \mathrm{~m}$. This value is optically set by the size of the Lyot stop in the camera and is consistent with the design expectations from Fowler et al. (2007). ${ }^{1}$

An analysis of the optical efficiency of the telescope gave $\eta_{A} / \eta_{m}=72.3 \pm 4.8 \%$. This is consistent with simulations, being dominated by diffraction effects (taper effects), detector pixel size and surface errors on the mirrors.

The accuracy of our measurements was limited by interference effects produced by the co-existence of multiple paths from the source to the focal plane. These multiple paths were produced by reflexions in parts of the inner structure of the telescope given the wide field of view of the source.

\section{ACKNOWLEDGEMENTS}

This project was partially funded by a FONDECYT Iniciación $\mathrm{N}^{\circ} 11100147$ award, a VRI Inicio 39/2010 award and FONDAP. We thank Lyman Page (Princeton University), for lending us the Gunn oscillator and chopper used, and for many suggestions and guiding. We also thank Juan Carlos Fluxa and Sergio Rojas for their collaboration in the project. We specially thank Masao Uehara for his help in performing the experiment at the site. We thank Parque Astronómico Atacama (CONICYT), in which the ACT telescope is located. We thank the Electrical Engineering department at PUC for letting us use the robotic laboratory to do the source characterization.

\section{REFERENCES}

[1] Fowler, J. W., Niemack, M. D., Dicker, S. R., Aboobaker, A. M., Ade, P. A. R., Battistelli, E. S., Devlin, M. J., Fisher, R. P., Halpern, M., Hargrave, P. C., Hincks, A. D., Kaul, M., Klein, J., Lau, J. M., Limon, M., Marriage, T. A., Mauskopf, P. D., Page, L., Staggs, S. T., Swetz, D. S., Switzer, E. R., Thornton, R. J., and 
Tucker, C. E., "Optical design of the Atacama Cosmology Telescope and the Millimeter Bolometric Array Camera," Applied Optics 46(17), 3444-3454 (2007).

[2] Swetz, D. S., Ade, P. A. R., Amiri, M., Appel, J. W., Battistelli, E. S., Burger, B., Chervenak, J., Devlin, M. J., Dicker, S. R., Doriese, W. B., Dünner, R., Essinger-Hileman, T., Fisher, R. P., Fowler, J. W., Halpern, M., Hasselfield, M., Hilton, G. C., Hincks, A. D., Irwin, K. D., Jarosik, N., Kaul, M., Klein, J., Lau, J. M., Limon, M., Marriage, T. A., Marsden, D., Martocci, K., Mauskopf, P., Moseley, H., Netterfield, C. B., Niemack, M. D., Nolta, M. R., Page, L. A., Parker, L., Staggs, S. T., Stryzak, O., Switzer, E. R., Thornton, R., Tucker, C., Wollack, E., and Zhao, Y., "Overview of the Atacama Cosmology Telescope: Receiver, Instrumentation, and Telescope Systems," Astrophysical Journal, Supplement 194, 41-+ (June 2011).

[3] Sunyaev, R. A. and Zel'dovich, Y. B., The Spectrum of Primordial Radiation, its Distortions and their Significance," Comments on Astrophysics and Space Physics 2, 66 (Mar. 1970).

[4] Hincks, A. D., Acquaviva, V., Ade, P. A. R., Aguirre, P., Amiri, M., Appel, J. W., Barrientos, L. F., Battistelli, E. S., Bond, J. R., Brown, B., Burger, B., Chervenak, J., Das, S., Devlin, M. J., Dicker, S. R., Doriese, W. B., Dunkley, J., Dünner, R., Essinger-Hileman, T., Fisher, R. P., Fowler, J. W., Hajian, A., Halpern, M., Hasselfield, M., Hernández-Monteagudo, C., Hilton, G. C., Hilton, M., Hlozek, R., Huffenberger, K. M., Hughes, D. H., Hughes, J. P., Infante, L., Irwin, K. D., Jimenez, R., Juin, J. B., Kaul, M., Klein, J., Kosowsky, A., Lau, J. M., Limon, M., Lin, Y.-T., Lupton, R. H., Marriage, T. A., Marsden, D., Martocci, K., Mauskopf, P., Menanteau, F., Moodlev, K., Moseley, H., Netterfield, C. B., Niemack, M. D., Nolta, M. R., Page, L. A., Parker, L., Partridge, B., Quintana, H., Reid, B., Sehgal, N., Sievers, J., Spergel, D. N., Staggs, S. T., Stryzak, O., Swetz, D. S., Switzer, E. R., Thornton, R., Trac, H., Tucker, C., Verde, L., Warne, R., Wilson, G., Wollack, E., and Zhao, Y., "The Atacama Cosmology Telescope (ACT): Beam Profiles and First SZ Cluster Maps," Astrophysical Journal, Supplement 191, 423-438 (Dec. 2010).

[5] Marriage, T. A., Acquariva, V., Ade, P. A. R., Aguirre, P., Amiri, M., Appel, J. W., Barrientos, L. F., Battistelli, E. S., Bond, J. R., Brornn, B., Burger, B., Chervenak, J., Das, S., Devlin, M. J., Dicker, S. R., Bertrand Doriese, W., Dunkley, J., Dünner, R., Essinger-Hileman, T., Fisher, R. P., Fowler, J. W., Hajian, A., Halpern, M., Hasselfield, M., Hernández-Monteagudo, C., Hilton, G. C., Hilton, M., Hincks, A. D., Hlozek, R., Huffenberger, K. M., Handel Hughes, D., Hughes, J. P., Infante, L., Irwin, K. D., Baptiste Juin, J., Kaul, M., Klein, J., Kosowsky, A., Lau, J. M., Limon, M., Lin, Y.-T., Lupton, R. H., Marsden, D., Martocci, K., Mauskopf, P., Menanteau, F., Moodley, K., Moseley, H., Netterfield, C. B., Niemack, M. D., Nolta, M. R., Page, L. A., Parker, L., Partridge, B., Quintana, H., Reese, E. D., Reid, B., Sehgal, N., Sherwin, B. D., Sievers, J., Spergel, D. N., Staggs, S. T., Swetz, D. S., Switzer, E. R., Thornton, R., Trac, H., Tucker, C., Warne, R., Wilson, G., Wollack, E., and Zhao, Y., "The Atacama Cosmology Telescope: Sunyaev-Zel'dovich-Selected Galaxy Clusters at $148 \mathrm{GHz}$ in the 2008 Survey," Astrophysical Journal 737, $61-+$ (Aug. 2011).

[6] Fowler, J. W., Acquaviva, V., Ade, P. A. R., Aguirre, P., Amiri, M., Appel, J. W., Barrientos, L. F., Battistelli, E. S., Bond, J. R., Brown, B., Burger, B., Chervenak, J., Das, S., Devlin, M. J., Dicker, S. R., Doriese, W. B., Dunkley, J., Dünner, R., Essinger-Hileman, T., Fisher, R. P., Hajian, A., Halpern, M., Hasselfield, M., Hernández-Monteagudo, C., Hilton, G. C., Hilton, M., Hincks, A. D., Hlozek, R., Huffenberger, K. M., Hughes, D. H., Hughes, J. P., Infante, L., Irwin, K. D., Jimenez, R., Juin, J. B., Kaul, M., Klein, J., Kosowsky, A., Lau, J. M., Limon, M., Lin, Y., Lupton, R. H., Marriage, T. A., Marsden, D., Martocci, K., Mauskopf, P., Menanteau, F., Moodley, K., Moseley, H., Netterfield, C. B., Niemack, M. D., Nolta, M. R., Page, L. A., Parker, L., Partridge, B., Quintana, H., Reid, B., Sehgal, N., Sievers, J., Spergel, D. N., Staggs, S. T., Swetz, D. S., Switzer, E. R., Thornton, R., Trac, H., Tucker, C., Verde, L., Warne, R., Wilson, G., Wollack, E., and Zhao, Y., "The Atacama Cosmology Telescope: A Measurement of the $600<\ell<8000$ Cosmic Microwave Background Power Spectrum at $148 \mathrm{GHz}$," Astrophysical Journal 722, 1148-1161 (Oct. 2010).

[7] Das, S., Marriage, T. A., Ade, P. A. R., Aguirre, P., Amiri, M., Appel, J. W., Barrientos, L. F., Battistelli, E. S., Bond, J. R., Brown, B., Burger, B., Chervenak, J., Devlin, M. J., Dicker, S. R., Bertrand Doriese, W., Dunkley, J., Dünner, R., Essinger-Hileman, T., Fisher, R. P., Fowler, J. W., Hajian, A., Halpern, M., Hasselfield, M., Hernández-Monteagudo, C., Hilton, G. C., Hilton, M., Hincks, A. D., Hlozek, R., Huffenberger, K. M., Hughes, D. H., Hughes, J. P., Infante, L., Irwin, K. D., Baptiste Juin, J., Kaul, M., Klein, J., Kosowsky, A., Lau, J. M., Limon, M., Lin, Y.-T., Lupton, R. H., M.Jarsden, D., Martocci, K., Mauskopf, 
P., Menanteau, F., Moodley, K., Moseley, H., Netterfield, C. B., Niemack, M. D., Nolta, M. R., Page, L. A., Parker, L., Partridge, B., Reid, B., Sehgal, N., Sherwin, B. D., Sievers, J., Spergel, D. N., Staggs, S. T., Swetz, D. S., Switzer, E. R., Thornton, R., Trac, H., Tucker, C., Warne, R., Wollack, E., and Zhao, Y., "The Atacama Cosmology Telescope: A Measurement of the Cosmic Microwave Background Power Spectrum at 148 and $218 \mathrm{GHz}$ from the 2008 Southern Survey," Astrophysical Journal 729, 62-+ (Mar. 2011).

[8] Dunkley, J., Hlozek, R., Sievers, J., Acquaviva, V., Ade, P. A. R., Aguirre, P., Amiri, M., Appel, J. W., Barrientos, L. F., Battistelli, E. S., Bond, J. R., Brown, B., Burger, B., Chervenak, J., Das, S., Devlin, M. J., Dicker, S. R., Bertrand Doriese, W., Dünner, R., Essinger-Hileman, T., Fisher, R. P., Fowler, J. W., Hajian, A., Halpern, M., Hasselfield, M., Hernández-Mfonteagudo, C., Hilton, G. C., Hilton, M., Hincks, A. D., Huffenberger, K. M., Hughes, D. H., Hughes, J. P., Infante, L., Irwin, K. D., Juin, J. B., Kaul, M., Klein, J., Kosowsky, A., Lau, J. M., Limon, M., Lin, Y.-T., Lupton, R. H., Marriage, T. A., Marsden, D., Mauskopf, P., Menanteau, F., Moodley, K., Moseley, H., Netterfield, C. B., Niemack, M. D., Nolta, M. R., Page, L. A., Parker, L., Partridge, B., Reid, B., Sehgal, N., Sherwin, B., Spergel, D. N., Staggs, S. T., Swetz, D. S., Switzer, E. R., Thornton, R., Trac, H., Tucker, C., Warne, R., Wollack, E., and Zhro, Y., "The Atacama Cosmology Telescope: Cosmological Parameters from the 2008 Power Spectrum," Astrophysical Journal 739, $52-+$ (Sept. 2011).

[9] Holland, W., Duncan, W', and Griffin, M., "Bolometers for Submillimeter and Millimeter Astronomy," in [Single-Dish Radio Astronomy: Techniques and Applications], Stanimirovic, S., Altschuler, D., Goldsmith, P., and Salter, C., eds., Astronomical Society of the Pacific Conference Series 278, 463-491 (Dec. 2002). 